\section{Revised guidelines for cardiovascular risk management}

With interest we read the study by van Duijn et al regarding the revised Dutch guidelines for cardiovascular risk management. ${ }^{1}$ The authors conducted a practice-based intervention study to explore the feasibility and consequences of a re-evaluation programme for patients without target organ damage, who were treated for hypertension and/or hypercholesterolemia under the previous guidelines. After reading their conclusions some questions remained.

First, the authors conclude that stopping medication in this group of patients can be assumed to be safe, as no important adverse effects such as heart failure or cardiovascular events took place during the 6-month follow-up. Considering the relatively small group of patients, short follow-up time, and lack of an appropriate control group, we doubt such a conclusion can be drawn based on these results. We feel that a more appropriate outcome regarding safety is the effect of discontinuation of medication on blood pressure and lipid profile. Although some results regarding these parameters are mentioned in the text, the authors, unfortunately, fail to present the full baseline and follow-up data of the participants.

Second, the authors present the finding that $10 \%$ of patients initially selected for reevaluation have discontinued their medication after 6 months. They go on to suggest that the efficiency of this reevaluation programme could be improved by initially focusing on young people and nonsmokers, as this group of patients was most likely to discontinue medication. However, the reason this group stopped most often appears to be based solely on the fact that GPs were most likely to advise this group to stop. The authors did not specify whether the GPs were instructed in giving advice in advance and whether they registered their decision before discussing their advice with the patient. We would be interested to know more about this aspect of the study methodology, as it may help to elucidate why this group in particular was advised to stop most often. Of those patients who were advised to stop medication, $56 \%$ had still discontinued their medication after 6 months. Perhaps focusing on the role of the GP within this re-evaluation programme would improve efficiency far more effectively than targeting specific patient groups.

We would be interested in the authors thoughts on these comments.

Marijn E Rigter,

Department of General Practice, University Medical Centre Rotterdam, the Netherlands.

Aafke Koffeman,

MD, Department of General Practice, Erasmus MC, University Medical Centre Rotterdam, PO Box 2040, 3000 CA, Rotterdam, the Netherlands.

E-mail address: a.koffemanderasmusmc.nl

\section{REFERENCE}

1. Van Duijn HJ, Belo JN, Blom JW, et al. Revised guidelines for cardiovascular risk management time to stop medication? Br J Gen Pract 2011; DOI: 10.3399/bjgp11X578025.

\section{DOI: 10.3399/bjgp11X593767}

\section{Authors' response}

We thank Rigter and Koffeman ${ }^{1}$ for their comments on our paper. ${ }^{2}$ The aim of this study was to explore the feasibility and consequences of a re-evaluation programme for patients without target organ damage who were treated for hypertension and/or hypercholesterolemia in general practices.

We have shown that stopping medication of itself can be assumed to be safe, as no important adverse effects took place during the 6-month follow-up. As stated in the discussion section of our paper, we agree that until now it is not known what the morbidity and mortality will be in the discontinuing versus the continuing group in the next 10 years. However, if pretreatment levels of blood pressure and cholesterol were unknown, we have chosen to assume high levels of systolic blood pressure $(180 \mathrm{mmHg})$ and total cholesterol to HDL-cholesterol ratio (8.0) to minimise the number of underestimated levels. Therefore, even if the blood pressure or lipid profile would change during follow-up, it would not alter the estimated low risk of cardiovascular mortality. Consequently, presenting data on these variables would thus have no additional value. Further, as also mentioned in the discussion section, the new guidelines on cardiovascular risk management are based on extensive clinical evidence. Therefore, we assume that stopping superfluous medication in patients with a low risk of cardiovascular mortality will not, of itself, increase mortality rate.

With respect to the efficiency of this reevaluation programme, we agree that the views of the GPs, nurse practitioners, and patients on discontinuing preventive cardiovascular medication seem to play a role in attendance and advising whether or not to stop medication, and restarting medication after withdrawal. For this reason, we have recommended in our paper further exploration of these views to enable better information to be given to patients and to overcome differences between GPs and nurse practitioners. This year, a cluster-randomised controlled noninferiority trial to the cost-effectiveness of proactive implementation of the multidisciplinary guideline cardiovascular risk management versus usual care in currently treated patients with hypertension and hypercholesterolemia will be launched as a follow-up of our study. However, until the results of this new study are presented, based on our results and calculation in the discussion section, it seems useful to focus on young people and non-smokers!

Huug J van Duijn,

Health Cooperative Katwijk langs de Rijn, Molenweg 16, Katwijk, 2223 HD, the Netherlands. E-mail: hjidvduijn.nl

Janneke N Belo,

Health Cooperative Katwijk langs de Rijn, Katwijk, the Netherlands.

\section{REFERENCES}

1. Koffeman A, Rigter M. Revised guidelines for cardiovascular risk management [letter]. Br J Gen Pract 2011; 61(590): 548

2. Van Duijn HJ, Belo JN, Blom JW, et al. Revised guidelines for cardiovascular risk management time to stop medication? Br J Gen Pract 2011; DOI: 10.3399/bjgp11X578025.

DOI: 10.3399/bjgp11X593776 